\title{
EVN observations of the methanol masers in Cep A
}

\author{
Karl Torstensson* \\ Leiden Observatory/JIVE \\ E-mail: kalleestrw.leidenuniv.nl \\ Huib Jan van Langevelde \\ JIVE/Leiden Observatory \\ E-mail: langevelde@jive.nl

\section{Wouter Vlemmings} \\ Argelander-Institut fuer Astronomie \\ E-mail: woutereastro.uni-bonn.de
}

\section{Floris van der Tak}

Netherlands Institute for Space Research (SRON)

E-mail: vdtakesron.n]

\begin{abstract}
We present EVN observations of the $6.7 \mathrm{GHz}$ methanol maser in the high mass star-forming region Cepheus A. The maser emission arises in a filamentary structure over $\sim 1200 \mathrm{AU}$, straddling the waist of the thermal jet HW2. The velocity field of the methanol masers shows an infall signature rather than a rotation signature. We present a model in which the methanol maser emission arises in a shock interface between the infalling gas and the accretion disk/process. The model is supported by the fact that we do not see any maser emission from the rear side of the ring which is shielded by the ionised gas in the thermal jet. We also present preliminary results of JCMT observations of thermal methanol in Cep A. The large scale molecular outflow provides further constrains on the geometry.
\end{abstract}

The 9th European VLBI Network Symposium on The role of VLBI in the Golden Age for Radio Astronomy and EVN Users Meeting

September 23-26, 2008

Bologna, Italy

\footnotetext{
*Speaker.
} 


\section{Introduction}

Methanol masers are a promising tool for the study of high-mass star-formation. Continuum emission of warm dust at sub-millimetre wavelengths has been detected at well over $95 \%$ of the observed $6.7 \mathrm{GHz}$ methanol maser sites [1], even though only some of the masers are associated with a detectable ultra-compact (UC) HII region. This seems to indicate that the masers probe a range of early phases of massive star formation and that the methanol maser emission disappears when the UC HII region is created [2]. More than just signposts, masers are fantastic tracers of the geometry and small scale dynamics of these regions. Much effort has focused on studying the kinematics and several claims of circum-stellar disks, expanding spherical shells and jets have been argued [3]-5]. The evidence suggest that masers trace an evolutionary sequence or possibly a mass range in the formation process, or perhaps even both.

The star forming region Cepheus A East is one of the most nearby high-mass star-forming regions at a distance of $700 \mathrm{pc}$ [6]. The region has a total bolometric luminosity of $2.5 \cdot 10^{4} \mathrm{~L} \odot$ [ []] and its appearance in the radio is dominated by the thermal jet HW2 [8]. A central object has been identified, which is believed to be the source driving the outflow. The outflow shows velocities in excess of $500 \mathrm{~km} \mathrm{~s}^{-1}$ [9]. Recent sub-millimetre observations have shown an elongated disk structure [10, 11] at the position of the $7 \mathrm{~mm}$ continuum object identified by [9]. Maser observations of the object have shown a complicated structure in $\mathrm{OH}$ [12], $\mathrm{H}_{2} \mathrm{O}$ [13], and $12 \mathrm{GHz} \mathrm{CH}_{3} \mathrm{OH}$ masers [14].

We have carried out large field of view EVN observations of the $6.7 \mathrm{GHz}$ methanol maser in Cep A. Additionally, as part of an ongoing project to map the distribution and excitation of the methanol gas in this object, we have done large scale observations of the $\mathrm{CH}_{3} \mathrm{OH}$ 7-6 band at $338.4 \mathrm{GHz}$ with the JCMT.

\section{Observations}

\subsection{Maser observations}

The observations were carried out in November 2004 with 8 telescopes of the EVN. Cep A was observed for $\sim 2 \mathrm{~h}$ including calibrators, resulting in $\sim 45 \mathrm{~min}$ on source. The observations were split into two $1 \mathrm{~h}$ blocks, separated by a $\sim 12 \mathrm{~h}$ to improve the uv-coverage. The experiment was setup at a centre frequency of $6668.5142 \mathrm{MHz}$ (rest frequency) with $2 \mathrm{MHz}$ bandwidth and 1024 channels, resulting in a velocity resolution of $\sim 0.1 \mathrm{~km} \mathrm{~s}^{-1}$. Both RCP and LCP were recorded. The data was correlated with the EVN correlator at JIVE using an integration time of $0.25 \mathrm{~s}$.

All calibration and imaging was done in AIPS. Amplitude and bandpass calibration was done on 3C345 and DA193, observed at the beginning and end of each block. For phase calibration and initial self-calibration J2302+6405 was observed every three minutes. The brightest maser feature was then used for further self-calibration, resulting in an rms of $\sim 3.5 \mathrm{mJy} \mathrm{beam}^{-1}$ in the emission free channels.

\subsection{JCMT observations}

The JCMT observation of Cep A were performed on June 2 and June 17, 2007. We used the array receiver HARP, which has 16 receptors with a spacing of $\sim 30^{\prime \prime}$ in a $4 \times 4$ grid, resulting in 
a footprint of $\sim 2^{\prime}$. We used the observing mode HARP5, a type of beam-switching (jiggle-chop) mode where several short scans at different positions of the target source (10 s per jiggle position) are observed before switching to the off position. The observing mode results in a map of 20x20 pixels covering $2^{\prime} \times 2^{\prime}$, thus each pixel is $6^{\prime \prime} \times 6^{\prime \prime}$. This ensures proper Nyquist sampling of the $14^{\prime \prime}$ telescope beam at $338 \mathrm{GHz}$. The ACSIS correlator backend was set up with a $1 \mathrm{GHz}\left(880 \mathrm{~km} \mathrm{~s}^{-1}\right)$ bandwidth and 2048 channels centered on the $\mathrm{CH}_{3} \mathrm{OH} 7(01)-6(01)$ line at $338.41 \mathrm{GHz}$. Data reduction was done using standard single dish procedures.

\section{Results}

\subsection{Maser results}

The $6.7 \mathrm{GHz}$ methanol maser emission in Cepheus A originates in an extended 1.7" (1200 AU) filamentary, arc-like structure, straddling the waist of the radio-jet HW2, Figure 1. The maser emission arises in 8 elongated maser spots separated spatially and/or in velocity. The individual maser features are elongated in the direction of the overall structure, strongly suggesting that the methanol maser in Cep A is originating in one, large scale structure. There is a small velocity gradient of $\sim 2.5 \cdot 10^{-3} \mathrm{~km} \mathrm{~s}^{-1} / \mathrm{AU}$ across the whole structure with higher velocities towards the centre.

In general the $6.7 \mathrm{GHz}$ and $12.2 \mathrm{GHz}$ methanol masers observed by [14] show a close association. The $6.7 \mathrm{GHz}$ methanol maser positions to the West correspond well to the $12.2 \mathrm{GHz}$ methanol masers observed by [14]. To the East the positional agreement is poorer, this could be an effect of the different epochs of observations; the spatial offset of $\sim 40$ mas and the epoch difference of 5.8 years imply a speed of $\sim 22 \mathrm{~km} \mathrm{~s}^{-1}$. In general, all the positions of the $6.7 \mathrm{GHz}$ and the $12.2 \mathrm{GHz}$ masers show similar velocities.

In only one position does the methanol maser emission in Cep A show any association to other masing species $\left(\mathrm{H}_{2} \mathrm{O}\right.$ or $\left.\mathrm{OH}\right)$, namely in the elongated structure to the East. The $\mathrm{CH}_{3} \mathrm{OH}$ maser

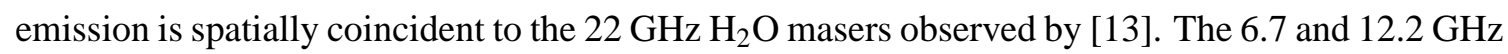
methanol masers are centred at $-4.2 \mathrm{~km} \mathrm{~s}^{-1}$. In contrast the $22 \mathrm{GHz} \mathrm{H}_{2} \mathrm{O}$ masers are observed at $\sim-14 \mathrm{~km} \mathrm{~s}^{-1}$.

The $6.7 \mathrm{GHz}$ methanol maser emission at the centre of Figure 1 (resolved into a few small spots) is projected against the thermal jet seen in the radio continuum. It is however not coincident with the $7 \mathrm{~mm}$ continuum object identified by [9] as the driving source but found further to the SW along the axis of the jet.

\subsection{Thermal methanol results}

Figure 2 shows the spatial distribution of the flux, the central velocity, and the width which we measure on the $\mathrm{CH}_{3} \mathrm{OH} 7(17)-7(16) \mathrm{E}$ line, which is the brightest un-blended feature in the JCMT spectra. The maps are centred on HW2 and, as can clearly be seen in the maps, both the flux and the line width peaks in the centre of the map close to the location of the $\mathrm{CH}_{3} \mathrm{OH}$ maser. In the velocity map we see the large scale outflow to the NE (blue-shifted) and SW (red-shifted), with a position angle of $\sim 60^{\circ}$. 


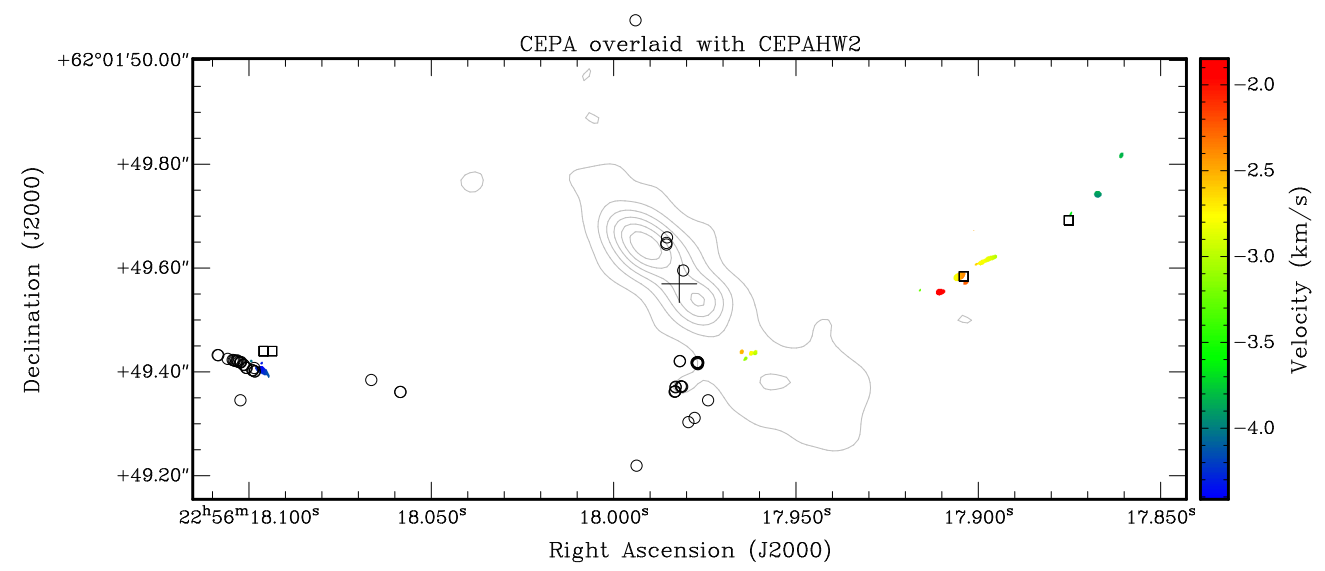

Figure 1: The velocity field of the $6.7 \mathrm{GHz} \mathrm{CH}_{3} \mathrm{OH}$ masers (colour) overlaid on $\mathrm{K}$ band continuum (con-

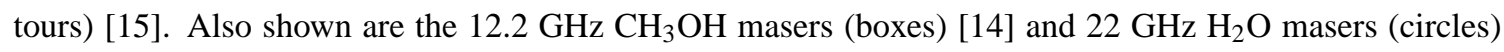
[13]. The position of the proto-star is indicated by the plus sign [9].
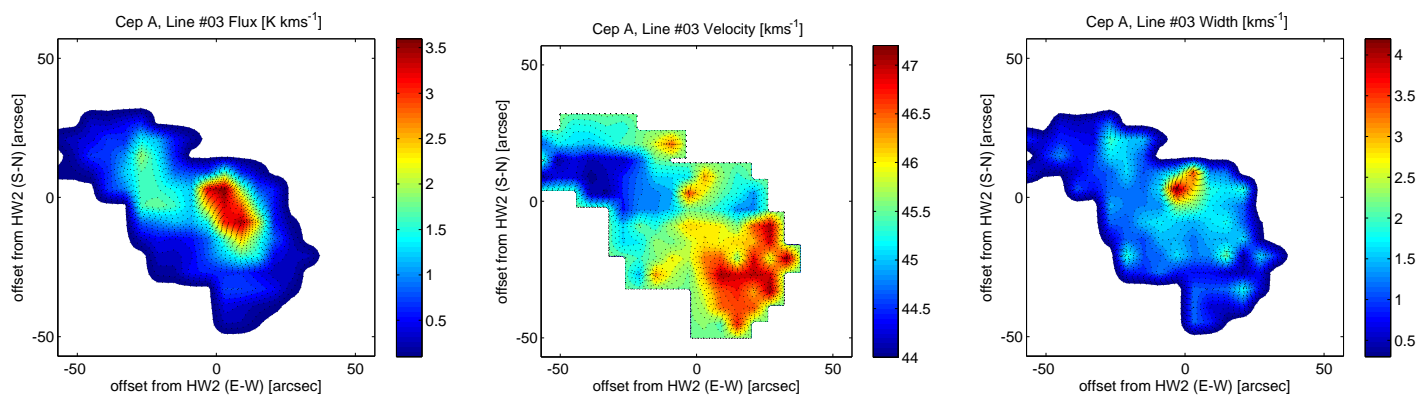

Figure 2: Integrated flux, velocity field, and line width of the $\mathrm{CH}_{3} \mathrm{OH} 7(17)-7(16)$ E-type transition, the strongest un-blended line observed with the JCMT. The figures are centred on the position of HW2. Note the blue-shifted molecular outflow to the NE and the red-shifted outflow to the SW.

\section{Analysis}

The methanol maser emission seems to arise in a large scale, ring-like structure close to or in the equatorial region of the high-mass object driving the HW2 jet. Such elliptical structures have recently been discovered in a substantial fraction of methanol masers studied in detail with VLBI [5][16]. To model this we have fitted an ellipse to the positions of our methanol maser spots. We solve for the ellipse's semi-major and semi-minor axis, the position angle and for the position offset with respect to the $7 \mathrm{~mm}$ continuum object identified by [9]. We then convert this fit to a position angle of the axis, a radius and inclination, assuming a circular ring structure. The best fit and model results are presented in Figure 3. As can clearly be seen the maser spots are consistent with an ellipse only slightly offset from the $7 \mathrm{~mm}$ continuum object. The radius of the fitted ring is 865 mas (605 AU). We find the position angle of the axis to be of $15.1^{\circ}$. This is in the same general direction as the position angle of the jet, $45^{\circ}$ [9] and the thermal outflow (Fig 2). The inclination of the ring is $74.9^{\circ}$, which is slightly larger than the best fit of $62^{\circ}$ that was found for the disk [10]. 

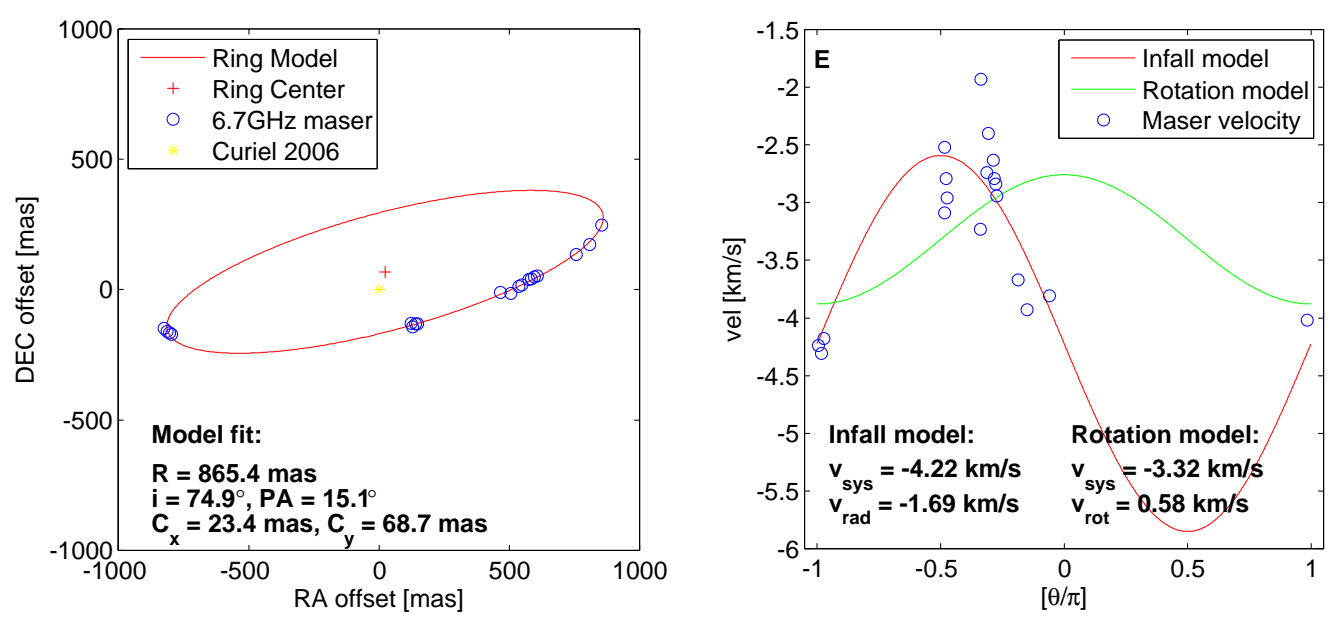

Figure 3: Fit of the ring model: Left panel shows the measured position in blue circles and the fitted ellipse in red. The yellow cross indicates the position of the $7 \mathrm{~mm}$ continuum object identified by [9]. Right panel shows in blue the measured velocities as a function of $\theta$ in our ring model. The red line shows the fit to a radial velocity model and the green line shows the result of a Keplerian velocity model.

Next, using the ellipse fitted to the methanol maser positions, we can compare the measured velocities to models that have different kinematic signatures: radial velocities or Keplerian rotation (Figure 3). For the radial velocity model we find a system velocity of $v_{s y s}=-4.2 \mathrm{~km} \mathrm{~s}^{-1}$ and a radial velocity $v_{\text {rad }}=-1.7 \mathrm{~km} \mathrm{~s}^{-1}$. For the rotation model we find a $v_{\text {sys }}=-3.3 \mathrm{~km} \mathrm{~s}^{-1}$ and a rotation velocity, $v_{r o t}=0.6 \mathrm{~km} \mathrm{~s}^{-1}$. Assuming Keplerian rotation this would correspond to a mass of $\sim 0.2 \mathrm{M}_{\odot}$, which is much too low for any reasonable model of the mass enclosed, therefore we rule out this possibility. Moreover, the fit is much poorer, with the high velocity emission observed towards the centre, whereas the outer regions show similar velocities. This is a sign of radial motions, not of rotation. Whether the radial motions can be attributed to infall or outflow, depends on the sign of the inclination; if the half-ellipse from which we see the maser emission is the front-side, then the signature is that of infall. Below we argue that most observed effects are indeed consistent with this.

\section{Discussion}

We argue that the axis of the ring is consistent with direction of the jet, as well as the large scale outflow seen in thermal methanol. The NE side of the outflow is blue-shifted (Figure 2, centre panel), and the maser emission in the centre of the image (seen in projection against the thermal jet) is located to the SW of the exciting object identified by [थ], this suggests the masers are on the near-side of the jet.

Moreover, the ionised gas in the thermal jet is expected to be optically thick at the frequency of $6.7 \mathrm{GHz}$, blocking at least some fraction of the far-side of the ring. This explains that we see mostly one side of the ring, the front.

We also note that the $6.7 \mathrm{GHz}$ and $12 \mathrm{GHz} \mathrm{CH} \mathrm{CH}_{3} \mathrm{OH}$ maser emission correspond closely both spatially and in velocity. But the $6.7 \mathrm{GHz} \mathrm{CH}_{3} \mathrm{OH}$ maser emission to the East that coincide spatially 
with the $22 \mathrm{GHz} \mathrm{H} \mathrm{H}_{2} \mathrm{O}$ maser emission is offset by $\sim 10 \mathrm{~km} \mathrm{~s}^{-1}$ in velocity. Possibly the methanol masers arise in a shock interface where the infalling gas is shocked before it reaches the accretion disk. The $\mathrm{CH}_{3} \mathrm{OH}$ masers occur in the pre-shocked gas whereas the $\mathrm{H}_{2} \mathrm{O}$ masers occur further downstream in the shock where the temperature and density are higher and the excitation conditions are more favourable for the $\mathrm{H}_{2} \mathrm{O}$ maser and less so for the $\mathrm{CH}_{3} \mathrm{OH}$ maser.

\section{Conclusions}

We find the $6.7 \mathrm{GHz} \mathrm{CH}_{3} \mathrm{OH}$ maser emission in Cep A to arise in a large scale ring structure straddling the waist of the thermal jet HW2. We have fitted a ring-model to our observations and find that the ring has a radius of 865 mas (605AU). Our HARP observations show that the molecular outflow to the NE is blue-shifted whereas the outflow to the SW is red-shifted. Because the axis angle of the ring is largely consistent with the ionised and molecular outflow, we argue that the ring is inclined with the South towards us. This implies that the masers are dominantly in front of the central exciting object, consistent with the observation of maser spots in front of the radio continuum. The velocity field therefore suggests an infall motion with a velocity of $1.7 \mathrm{~km} \mathrm{~s}^{-1}$. Because of the orientation of the ring structure and the large velocity offset between the methanol and water maser emission we speculate that the maser emission arises in a shock interface prior to the gas being entrained in an accretion disk.

\section{References}

[1] Hill, Burton, Minier, et al. 2005, MNRAS, 363, 405

[2] Walsh, Burton, Hyland, \& Robinson 1998, MNRAS, 301, 640

[3] Pestalozzi, Elitzur, Conway, \& Booth 2004, ApJ, 603, L113

[4] Minier, Booth, \& Conway 2002, A\&A, 383, 614

[5] Bartkiewicz, Szymczak, \& van Langevelde 2005, A\&A, 442, L61

[6] Moscadelli, Reid, Menten, et al. 2008, 2008arXiv0811.0679M

[7] Evans, Slovak, Becklin, et al. 1981, ApJ, 244, 115

[8] Hughes \& Wouterloot 1984, ApJ, 276, 204

[9] Curiel, Ho, Patel, et al. 2006, ApJ, 638, 878

[10] Patel, Curiel, Sridharan, et al. 2005, Nature, 437, 109

[11] Torrelles, Patel, Curiel, et al. 2007, ApJ, 666, L37

[12] Bartkiewicz, Szymczak, Cohen, \& Richards 2005, MNRAS, 361, 623

[13] Vlemmings, Diamond, van Langevelde, \& Torrelles 2006, A\&A, 448, 597

[14] Minier, Conway, \& Booth 2001, A\&A, 369, 278

[15] Torrelles, Gómez, Garay, et al. 1998, ApJ, 509, 262

[16] Bartkiewicz, Szymczak \& van Langevelde, Methanol masers survey using EVN, PoS(IX EVN Symposium) 037 